\title{
Effects of Eccentric Mold Electromagnetic Stirring on Continuous Casting Large Steel Round Blooms
}

\author{
Liang Niu ${ }^{1,2}$, Junxue Zhao ${ }^{1, *}$ and Shengtao Qiu $^{2}$ \\ ${ }^{1}$ School of Metallurgical Engineering, Xi an University of Architecture and Technology, Xi an 710055, Shaanxi China \\ ${ }^{2}$ National Engineering \& Research Center of Continuous Casting Technology, Beijing 100081, China
}

In this paper, a coupled 3D mathematical model is established to study the electromagnetic, flow and temperature fields of round blooms with different degrees of eccentric mold electromagnetic stirring (M-EMS). The results show that under the action of severely eccentric M-EMS, the magnetic flux density and time-averaged electromagnetic force near the external arc side of a $\Phi 380 \mathrm{~mm}$ round bloom are greater than those near the inner arc side; the inertial impingement jet from the nozzle is deflected toward the external arc sides, and the temperature of molten steel on the external arc side is higher. As the degree of M-EMS eccentricity decreases, the differences in the electromagnetic, flow and temperature fields between the inner and external arc sides of the round blooms gradually decrease. The molten steel temperature on the inner arc side increases significantly after moving the nozzle position to the inner arc side of the $\Phi 380 \mathrm{~mm}$ round bloom, so this method is not suitable to eliminate the effects of M-EMS eccentricity on $\Phi 380 \mathrm{~mm}$ round blooms. [doi:10.2320/matertrans.MT-M2019376]

(Received January 6, 2020; Accepted June 29, 2020; Published September 25, 2020)

Keywords: round bloom, mold electromagnetic stirring, eccentric stirring, nozzle position

\section{Introduction}

The large round blooms have been widely used in the manufacture of seamless steel pipes, and the wind power industry. ${ }^{1,2)}$ With the develop of relevant industries, the demands for large round blooms with high quality continue to increase. The mold electromagnetic stirring (M-EMS) has been proved to be the most extremely effective method for increasing the equiaxed crystal ratio (ECR) of the billet and alleviating macro-segregation. ${ }^{3-8)}$

In considerations of the cost and the time spend on replacing the M-EMS equipment, in the production process, the round blooms with different diameters are usually produced using the same M-EMS device. The installation position of the M-EMS is designed and fixed according to the maximum-diameter round bloom, and the positions of the external arc reference lines of round blooms with different diameters are the same, as shown in Fig. 1. This installation method results in the eccentricity of the cross-sections between the round bloom with smaller diameter and the M-EMS device, which leads to the asymmetry of the M-EMS effect in the round bloom. Researches ${ }^{9,10)}$ show that the seriously eccentric M-EMS makes the carbon content and inclusions near the external arc side of the round bloom significantly lower than that near the inner arc side, and these uneven distribution of carbon content imparts large differences to the mechanical properties of the round bloom. The basic principle of M-EMS is to improve the quality of round blooms by controlling the flow and heat transfer of molten steel. However, there is few researches focus on the effects of eccentric M-EMS on the flow and heat transfer of large round blooms.

The purpose of this paper is to numerically analyze the characteristic of the 3D electromagnetic, flow and temperature fields of $\Phi 380 \mathrm{~mm}, \Phi 500 \mathrm{~mm}$ and $\Phi 600 \mathrm{~mm}$ $38 \mathrm{CrMoAl}$ round blooms under the action of eccentric M-EMS. The results show that the temperature difference

*Corresponding author, E-mail: yejinjieneng@126.com

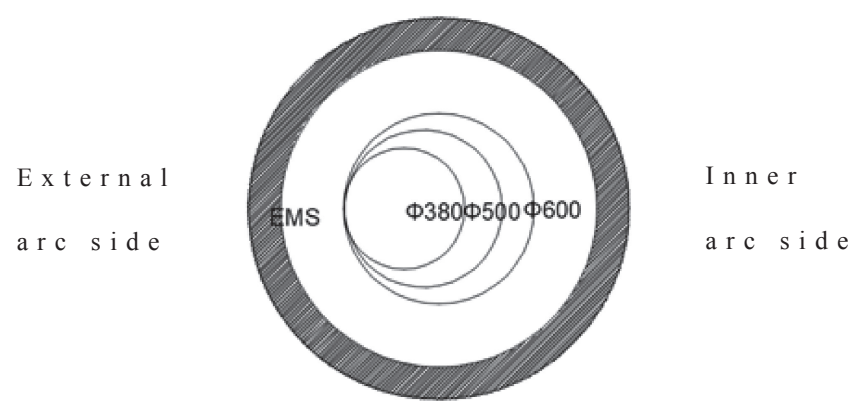

Fig. 1 Schematic illustrations of mold eccentric stirring.

between the inner and external arc sides of the $\Phi 380 \mathrm{~mm}$ round bloom is quite large. In order to reduce the influence of eccentric M-EMS, this paper attempts to adjust the nozzle position of $\Phi 380 \mathrm{~mm}$ round bloom.

\section{Numerical Simulation}

The direct study of the eccentric M-EMS in the continuous casting process is difficult since the opacity of the molten steel in the casting mold. In this paper, numerical simulation has been used as the basic method to investigate the effects of eccentric M-EMS on the electromagnetic, flow and temperature fields of round blooms. The basic parameters, 3-D model and physics formulas of numerical simulations are shown as follows.

\subsection{Basic parameters}

$38 \mathrm{CrMoAl}$ steel as one of alloy structural steels with high output and various applications, is one of the steel grades with the largest output in this steel plant, and its composition is shown in Table 1. The process parameters of $38 \mathrm{CrMoAl}$ round blooms with different diameters are listed in Table 2, and the $\Phi 380 \mathrm{~mm}, \Phi 500 \mathrm{~mm}$ and $\Phi 600 \mathrm{~mm}$ round blooms adopt the same M-EMS device. In actual production, it is easy to cause nozzle flocculation when the superheat of the molten steel is less than $20 \mathrm{~K}$ as a result of high viscosity 
Table 1 Chemical component of $38 \mathrm{CrMoAl} / \%$.

\begin{tabular}{cccccccccc}
\hline $\mathrm{C}$ & $\mathrm{Si}$ & $\mathrm{Mn}$ & $\mathrm{Cr}$ & $\mathrm{Mo}$ & $\mathrm{Al}$ & $\mathrm{P}$ & $\mathrm{S}$ & $\mathrm{Fe}$ \\
\hline 0.38 & 0.3 & 0.45 & 1.5 & 0.2 & $0.8-1$ & $<0.035$ & $<0.035$ & Bal.
\end{tabular}

Table 2 Process parameters of round blooms.

\begin{tabular}{|c|c|c|c|}
\hline Process parameters & $\Phi 380 \mathrm{~mm}$ & $\Phi 500 \mathrm{~mm}$ & $\Phi 600 \mathrm{~mm}$ \\
\hline Casting speed $(\mathrm{m} / \mathrm{min})$ & 0.65 & 0.35 & 0.28 \\
\hline $\mathrm{M}-\operatorname{EMS}(\mathrm{A} / \mathrm{Hz})$ & $400 / 3$ & $350 / 3$ & $300 / 2.5$ \\
\hline $\begin{array}{c}\text { Secondary cooling water } \\
\text { ratio }(\mathrm{L} / \mathrm{kg})\end{array}$ & 0.20 & 0.15 & 0.09 \\
\hline $\begin{array}{c}\text { Mold water flow ratio } \\
\qquad(\mathrm{L} / \mathrm{min})\end{array}$ & 3300 & 4000 & 4300 \\
\hline \multicolumn{3}{|c|}{$\begin{array}{l}\text { Temperature difference of between in } \\
\text { outlet water in mold (K) }\end{array}$} & $5-6$ \\
\hline \multicolumn{3}{|c|}{ Super heat $(K)$} & $20-30$ \\
\hline
\end{tabular}

caused by $\mathrm{Al}$ content of the molten steel, while when the superheat is higher than $30 \mathrm{~K}$, the ECR of the round bloom is small, and the macro-segregation of the round bloom is severe.

\subsection{Geometrical model}

The electromagnetic and the flow-temperature field are calculated with two kinds of software, and the geometric models need to be established separately. The electromagnetic field and the flow-temperature field of the eccentric M-EMS process are simulated using separated models. Figure 2(a) and (b) shows the geometric models of the magnetic field and the flow - temperature field of the $\Phi 600 \mathrm{~mm}$ round bloom, respectively. The coordinate system used for simulations is showed in Fig. 2(b), and the origin is set at the center of the mold exit plane, and the y coordinate axis is perpendicular to the external arc reference line. Threephase M-EMS is adopted in this paper, with an operating principle similar to a three-phase asynchronous electrical motor.
The calculated length of the round bloom is $1180 \mathrm{~mm}$. The copper mold height is $780 \mathrm{~mm}$ and its wall thickness is $30 \mathrm{~mm}$. The inner diameter of the straight-through nozzle is $40 \mathrm{~mm}$, while the SEN depth from the meniscus is $100 \mathrm{~mm}$. The iron core height of the stirrer is $300 \mathrm{~mm}$, and the bottom of the stirrer is at the same horizontal plane as the mold exit.

\subsection{Mathematical formulations}

The transport phenomena of M-EMS are results of the interactions among the magnetic, flow and temperature fields. Due to the complexity of the coupled simulation, the following assumptions have been made to make the problem easier to solve without losing its meaning: ${ }^{11)}$

(1) The molten steel is a homogeneous incompressible fluid, and the viscosity, specific heat, and thermal conductivity are assumed to be temperature independent.

(2) The effects of curvature and vibration of the mold on molten steel flow are ignored.

(3) The effects of the displacement current and flow field in the bloom on the magnetic field are ignored.

(4) The arc bending of the round bloom is ignored, and the heat transfer condition of the round bloom is symmetrical.

\subsubsection{Electromagnetic field}

When the low-frequency region is considered, the electromagnetic field can be simplified as a quasi-static magnetic field. The formulas relevant to M-EMS include the following:

Faraday's law:

$$
\nabla \times \boldsymbol{E}=-\partial \boldsymbol{B} / \partial \mathrm{t}
$$

Ampere's law:

$$
\nabla \times \boldsymbol{H}=\boldsymbol{J} \mu_{0} \mu_{\mathrm{r}}
$$

Gauss's law for magnetism:

$$
\nabla \times \boldsymbol{B}=0
$$

Gauss's law:

$$
\nabla \times \boldsymbol{D}=0
$$

Ohm's law:

$$
\boldsymbol{J}=\sigma \cdot \boldsymbol{E}
$$

where $\mathrm{B}$ is the magnetic flux density, $\mathrm{T}$; $\mathrm{J}$ is the current density, $\mathrm{A} / \mathrm{m}^{2} ; \mathrm{E}$ is the electric field strength, $\mathrm{V} / \mathrm{m} ; \sigma$ is the electrical conductivity, $\mathrm{S} / \mathrm{m}$; $\mathrm{t}$ is the time, $\mathrm{s} ; \mathrm{D}$ is the electric
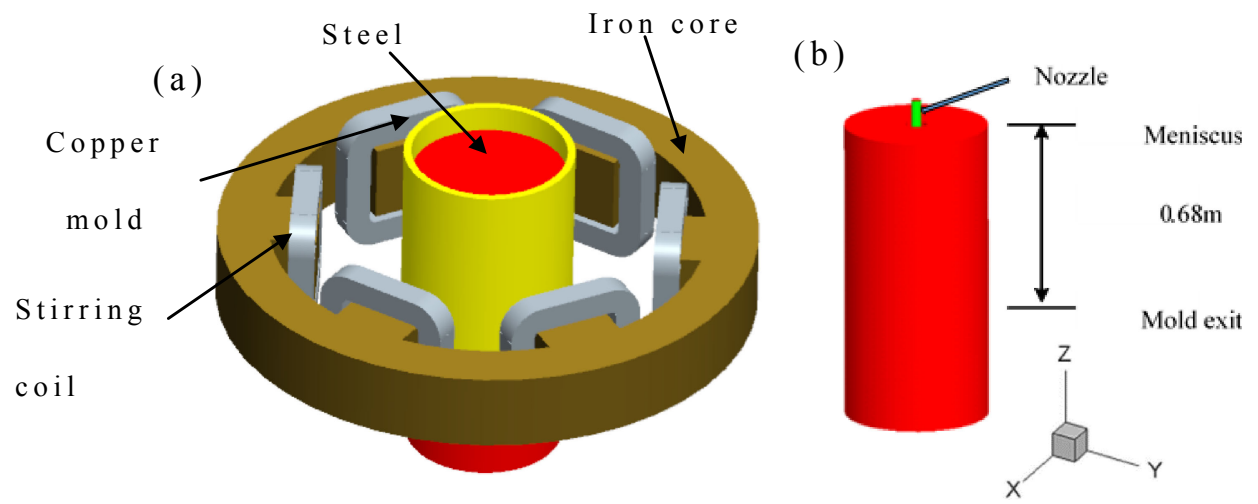

Fig. 2 Geometric models of the $\Phi 600 \mathrm{~mm}$ round bloom. (a) Magnetic field, (b) flow-Temperature field. 
displacement vector, $\mathrm{H}$ is the magnetic field intensity, $\mathrm{A} / \mathrm{m}$; $\mu_{0}$ is the magnetic permeability of the vacuum, and $\mu_{\mathrm{r}}$ is the relative permeability.

The electromagnetic force density is computed as follows:

$$
F_{\text {time }}=\boldsymbol{J} \times \boldsymbol{B}
$$

where $F_{\text {time }}$ is the transient electromagnetic force, $\mathrm{N} / \mathrm{m}^{3}$.

In the electromagnetic stirring process, the exciting current is sinusoidal and the electromagnetic field is harmonic. The electromagnetic force density changes over time. In this paper, two softwares are used to calculate the magnetic field and flow field respectively, and the electromagnetic force is loaded into the flow field calculation as a momentum source. The electromagnetic force data cannot be completely transmitted to the flow field calculation. The electromagnetic force generated by the M-EMS satisfies the sine function, so the time-average electromagnetic force can reflect the distribution of the electromagnetic force of the large round bloom in a period. ${ }^{11,12)}$ the time-average electromagnetic force is calculated as follows:

$$
\mathbf{F}_{\mathrm{ave}}=\frac{\boldsymbol{F}_{\mathbf{0}}+\boldsymbol{F}_{\mathbf{9 0}}}{2}
$$

where $F_{0}$ is the electromagnetic force at the initial moment, and $F_{90}$ is the electromagnetic force at the $1 / 4$ period.

\subsubsection{Fluid flow}

The continuity equation is as follows:

$$
\frac{\partial \cdot\left(\rho \boldsymbol{u}_{j}\right)}{\partial x_{j}}=0
$$

where $\rho$ is the density, $\mathrm{kg} / \mathrm{m}^{3} ; u_{j}$ is the velocity, $\mathrm{m} / \mathrm{s} ; x_{j}$ is the $\mathrm{j}$ spatial coordinate.

The momentum equation can be written as follows:

$$
\begin{aligned}
& \frac{\partial}{\partial \mathrm{t}}\left(\rho \boldsymbol{u}_{\boldsymbol{i}}\right)+\rho \cdot \frac{\partial\left(\boldsymbol{u}_{\boldsymbol{i}} \boldsymbol{u}_{j}\right)}{\partial x_{j}} \\
& \quad=-\frac{\partial \mathrm{p}}{\partial x_{i}}+\frac{\partial}{\partial x_{j}}\left[\mu_{e f f}\left(\frac{\partial \boldsymbol{u}_{\boldsymbol{i}}}{\partial x_{j}}+\frac{\partial \boldsymbol{u}_{j}}{\partial u_{i}}\right)\right]+\rho \boldsymbol{g}+\mathbf{F}_{\boldsymbol{a v e}}+\mathbf{S}_{\boldsymbol{p}}(9)
\end{aligned}
$$

where $\mathrm{p}$ is the pressure, $\mathrm{Pa} ; \mathrm{g}$ is the gravitational acceleration, $\mathrm{m} / \mathrm{s}^{2} ; \mathrm{F}_{\text {ave }}$ is the electromagnetic force, $\mathrm{N} / \mathrm{m}^{3}$; $\mu_{\text {eff }}$ is the effective viscosity coefficient determined by the $\mathrm{k}-\varepsilon$ turbulence model, $\mathrm{kg} /(\mathrm{m} \cdot \mathrm{s}) ; \mu_{l}$ and $\mu_{t}$ are respectively the physical viscosity coefficient and the turbulence viscosity.

$$
\mu_{\text {eff }}=\mu_{1}+\mu_{\mathrm{t}}
$$

The mushy zone is treated as a porous medium and the Darcy's law is applied. $S_{p}$, the Darcy source, can be written as follows: ${ }^{12)}$

$$
\boldsymbol{S}_{\boldsymbol{p}}=\frac{\left(1-f_{l}\right)}{f_{l}^{3}+0.001} \cdot A_{\text {mush }}\left(\boldsymbol{u}-\boldsymbol{v}_{\text {cast }}\right)
$$

$v_{\text {cast }}$ is the casting speed, $\mathrm{m} / \mathrm{s}$. $A_{\text {mush }}$ is the mushy zone constant, depending on the morphology of the solidifying alloy. The value of $A_{\text {mush }}$ must be large enough to force the fluid velocity equal to the casting speed when solidification and it is usually between $1 \times 10^{5}-1 \times 10^{8} .{ }^{12-14)}$ Many scholars have studied the value of $A_{\text {mush }}$, the results showed that the steel cooled down rapidly and the mold temperature remained unrealistically when the $A_{\text {mush }}$ at low value, and the result was deemed mostrealistic when $A_{m u s h}$ is $1 \times 10^{7}$, but the computation was difficult to get convergence. In some researches, the $A_{\text {mush }}$ was set to be $1 \times 10^{8}$ to obtain the accurate simulation result. ${ }^{11,15)} f_{l}$, the liquid fraction, can be written as follows:

$$
f_{l}=1-f_{s}=\left\{\begin{array}{cc}
1 & T \geq T_{l} \\
\frac{T-T_{s}}{T_{l}-T_{s}} & T_{s}<T<T_{l} \\
0 & T \leq T_{s}
\end{array}\right.
$$

where $T_{l}$ is the liquidus temperature, $\mathrm{K} ; T_{S}$ is solidus temperature, $\mathrm{K}$.

\subsubsection{Heat transfer}

The energy equation is as follows:

$$
\begin{gathered}
\frac{\partial}{\partial \mathrm{t}}(\rho \mathrm{H})+\rho \cdot \frac{\partial\left(\mathrm{u}_{j} \mathrm{H}\right)}{\partial \mathrm{x}_{j}}=\frac{\partial}{\partial \mathrm{x}_{j}}\left(k_{\text {eff }} \cdot \frac{\partial \mathrm{H}}{\partial \mathrm{x}_{i}}\right) \\
\mathrm{H}=h_{r e f}+\int_{\mathrm{T}_{r e f}}^{\mathrm{T}} \mathrm{C}_{\mathrm{p}} \mathrm{dT}+f_{l} \mathrm{~L}
\end{gathered}
$$

where $\mathrm{H}$ is the total enthalpy, $\mathrm{kJ} / \mathrm{kg} ; \mathrm{k}_{\text {eff }}$ is the effective thermal conductivity, $\mathrm{W} /(\mathrm{m} \cdot \mathrm{K}) ; \mathrm{C}_{\mathrm{p}}$ is the specific heat of molten steel. $\mathrm{J} /(\mathrm{kg} \cdot \mathrm{K})$ and $\mathrm{L}$ is the latent heat of molten steel, $\mathrm{J} / \mathrm{kg} ; h_{r e f}$ is the reference enthalpy, $\mathrm{kJ} / \mathrm{kg} ; \mathrm{T}_{\text {ref }}$ is the reference temperature, $\mathrm{K}$.

\subsection{Boundary and initial conditions \\ 2.4.1 Electromagnetic field}

Three-phase AC power is supplied to the coil windings for calculating the electromagnetic fields, and the boundary difference of each current phase is $120^{\circ}$. An air cylinder is used around the whole geometry for capturing the magnetic induction lines in the surrounding air. Magnetic flux parallel boundary conditions are applied on the external surfaces of the surrounding air cylinder.

\subsubsection{Fluid filed}

The free surface is specified as a zero-shear condition, and the velocity of the strand wall is specified as the casting speed.

For the computational inlet, the turbulent kinetic energy and turbulent dissipation rate can be determined by eqs. (15) and (16).

$$
\begin{gathered}
K=0.01 v_{\text {in }}^{2} \\
\varepsilon=K^{1.5} /\left(d_{0} / 2\right)
\end{gathered}
$$

where $v_{\text {in }}$ is the inlet velocity, $\mathrm{m} / \mathrm{s} ; \mathrm{d}_{0}$ is the nozzle diameter, $\mathrm{m}$.

The outlet of molten steel in the calculation domain is considered to be fully developed, and the derivative of any variable on the normal is 0 . For the free surface, the normal derivative of all variables is set to zero.

\subsubsection{Temperature field}

The inlet velocity is determined by casting speed according to the conservation of mass. The inlet temperature is the casting temperature of molten steel as follows:

$$
\mathrm{T}_{\text {in }}=\mathrm{T}_{1}
$$

where $\mathrm{T}_{\text {in }}$ is inlet temperature and $\mathrm{T}_{1}$ is the casting temperature, $\mathrm{K}$.

The adiabatic condition is adopted for the free surface. 
The heat transfer boundary conditions of the mold are divided into 2 parts: mold zone and foot roller zone. The heat flux of mold can be calculated by eq. (18): ${ }^{11)}$

$$
\bar{q}=\frac{C_{\mathrm{P}, \mathrm{N}} \cdot p_{\mathrm{w}} \cdot Q_{\mathrm{N}} \cdot \Delta T_{\mathrm{N}}}{F_{\mathrm{m}}}
$$

where $\bar{q}$ is the heat flux density, $\mathrm{W} / \mathrm{m}^{2} ; \mathrm{C}_{\mathrm{P}, \mathrm{N}}$ is the specific heat of cooling water, $\mathrm{J} / \mathrm{kg} /{ }^{\circ} \mathrm{C} ; \mathrm{Q}_{\mathrm{W}}$ is the cooling water flow rate of the mold, $\mathrm{m}^{3} / \mathrm{s} ; \Delta \mathrm{T}_{\mathrm{w}}$ is the temperature difference between cooling water inlet and outlet of the mold, ${ }^{\circ} \mathrm{C} ; \mathrm{F}_{\mathrm{m}}$ is the effective area of the mold, $\mathrm{m}^{2}$; and $\mathrm{p}_{\mathrm{w}}$ is the density of water, $\mathrm{kg} / \mathrm{m}^{3}$.

The heat transfer coefficient of the foot roller zone can be calculated by eq. $(19):^{16,17)}$

$$
\mathrm{h}_{f}=581 w^{0.541}
$$

where $h_{f}$ is the heat transfer coefficient, $\mathrm{W} /\left(\mathrm{m}^{2} \cdot \mathrm{K}\right)$; $\mathrm{w}$ is the water flow rate in the foot roller zone, $\mathrm{L} /\left(\mathrm{m}^{2} \mathrm{~s}\right)$.

The boundary conditions of round blooms with different diameters are listed in Table 3.

\subsection{Physical parameters of materials}

The physical parameters of materials are listed in Table 4.

\begin{tabular}{|c|c|c|c|}
\hline & $\Phi 380 \mathrm{~mm}$ & $\Phi 500 \mathrm{~mm}$ & $\Phi 600 \mathrm{~mm}$ \\
\hline $\begin{array}{c}\text { Inlet } \\
\text { ve } 1 \text { ocity }(\mathrm{m} / \mathrm{s})\end{array}$ & 0.978 & 0.911 & 1.05 \\
\hline $\mathrm{k}$ & 0.0096 & 0.0083 & 0.011 \\
\hline$\varepsilon$ & 0.047 & 0.038 & 0.058 \\
\hline $\begin{array}{c}\text { Casting } \\
\text { te mperature (K) }\end{array}$ & 1803 & 1803 & 1803 \\
\hline $\bar{q}\left(W / m^{2}\right)$ & $1.42 \times 10^{6}$ & $1.31 \times 10^{6}$ & $1.17 \times 10^{6}$ \\
\hline $\mathrm{h}_{f}\left(\mathrm{~W} \cdot \mathrm{m}^{-2} \cdot \mathrm{K}^{-1}\right)$ & 800 & 600 & 360 \\
\hline
\end{tabular}

Table 3 Boundary conditions of the round blooms.

Table 4 Physical parameters of the materials.

\begin{tabular}{cc}
\hline Parameter & Value \\
Density of steel & $7020 \mathrm{~kg} / \mathrm{m}^{3}$ \\
Specificheat of steel & $0.0064 \mathrm{~Pa} \cdot \mathrm{s}$ \\
Thermal conductivity of steel & $750(\mathrm{~J} /(\mathrm{kg} \cdot \mathrm{k}))$ \\
Magnetic permeability of vacuum & $35 \mathrm{~W} /(\mathrm{m} \cdot \mathrm{k})$ \\
Relative permeability of steel, & $26 \times 10^{-6} \mathrm{H} / \mathrm{m}$ \\
copper mold, stiring coil, and air & 1 \\
Iron core relative permeability & 1000 \\
Electrical conductivity of steel & $7.14 \times 10^{5} \mathrm{~S} / \mathrm{m}$ \\
Copper mold conductivity $(298 \mathrm{~K})$ & $4.7 \times 10^{7} \mathrm{~S} / \mathrm{m}$ \\
Copper mold conductivity $(500 \mathrm{~K})$ & $3.18 \times 10^{7} \mathrm{~S} / \mathrm{m}$ \\
Liquidus temperature of steel & $1773 \mathrm{~K}$ \\
Solidus temperature of steel & $1700 \mathrm{~K}$ \\
Latent heat of molten steel & $2700 \mathrm{~J} / \mathrm{kg}$ \\
Meltingtemperature of pure iron & $1811 \mathrm{~K}$ \\
\hline
\end{tabular}

\subsection{Numerical solution procedure}

The calculation of numerical modeling was divided into 3 steps: first, ANSYS-APDL software was used to calculate the magnetic field and electromagnetic force; secondly, the electromagnetic force density was loaded into momentum equation as source term by "user defined functions (UDF)" of the ANSYS-FLUENT; finally, the flow field and temperature field were calculated by ANSYS-FLUENT. Transient calculation is adopted to enhance the convergence stability. The adaptive time step is $0.01 \mathrm{~s}$, and the total calculating time is set as $350 \mathrm{~s}$.

\section{Results and Discussion}

\subsection{Electromagnetic fields}

Figure 3 demonstrates the magnetic flux density in the radiation direction of the mold of $\Phi 600 \mathrm{~mm}$ round bloom. The continuous curve is the simulation result, while the dotted points in figure are the measured magnetic flux density value using a Gauss meter. The results show that the simulation is in consistent with the reality magnetic field distribution in the mold. Note that the maximum magnetic flux density does not appear at the M-EMS mid-plane. This can be interpreted as the field shielding of the copper mold affecting the electromagnetic field distribution.

The magnetic flux density in the profile section of the round bloom $(t=0)$ are shown in Fig. 4. The magnetic flux density distribution of the $\$ 600 \mathrm{~mm}$ round bloom is symmetrical along its central axis, but the magnetic flux density distribution of the $\$ 380 \mathrm{~mm}$ and $\$ 500 \mathrm{~mm}$ round bloom is asymmetric, as a result of the eccentric placement of M-EMS device. Obviously, the magnetic flux density on the external arc side is larger than that on the inner arc side. The unevenness of magnetic field distribution becomes even serious with the decrease of the round bloom diameter.

The magnetic flux densities at the external and inner arc sides of the round blooms $(t=0)$ are shown in Fig. 5. Due to the different degrees of eccentric M-EMS, the maximum magnetic flux density on the inner arc side of $\Phi 500 \mathrm{~mm}$ and $\Phi 600 \mathrm{~mm}$ round blooms are located at the mold exit. But to

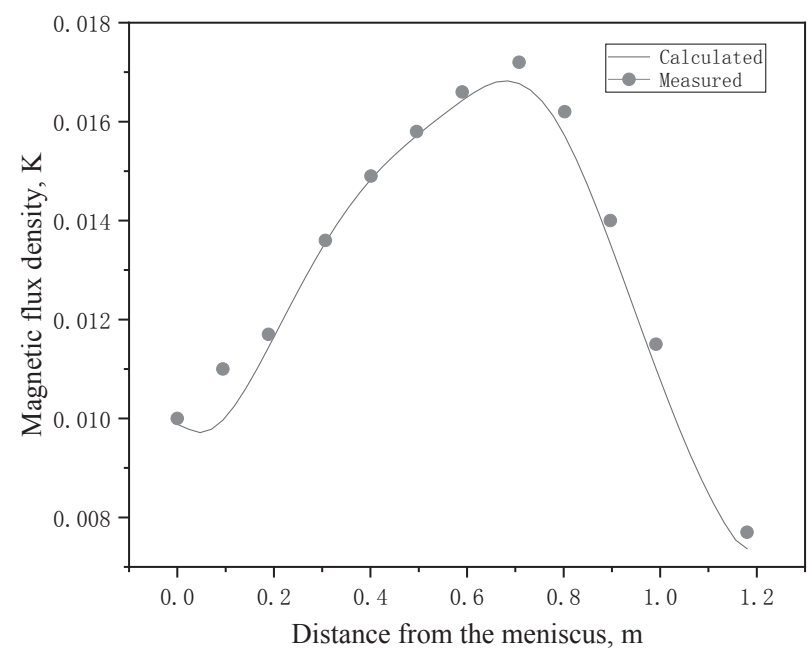

Fig. 3 Calculated and measured magnetic flux density along the strand center. 


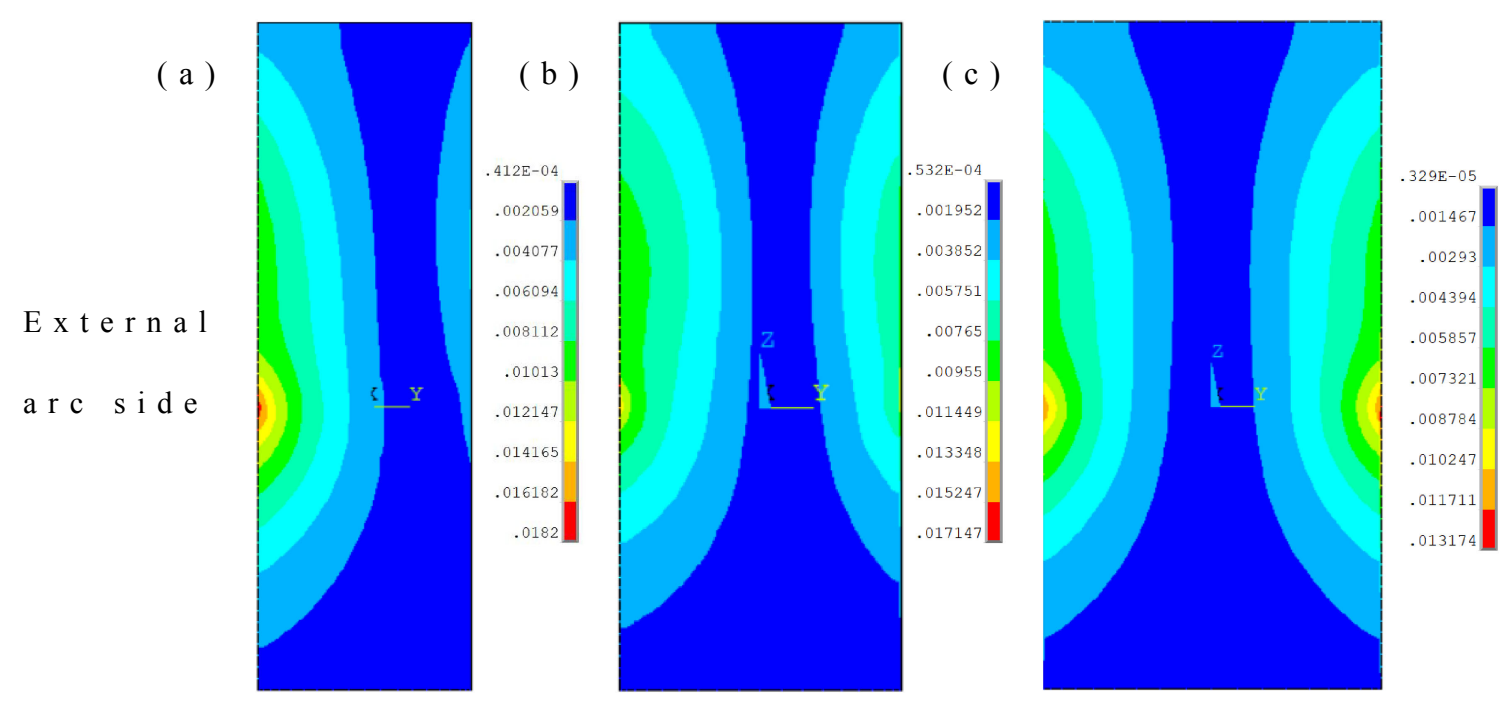

Fig. 4 Magnetic flux density in the profile sections (T). (a) $\Phi 380 \mathrm{~mm}$, (b) $\Phi 500 \mathrm{~mm}$, (c) $\Phi 600 \mathrm{~mm}$.

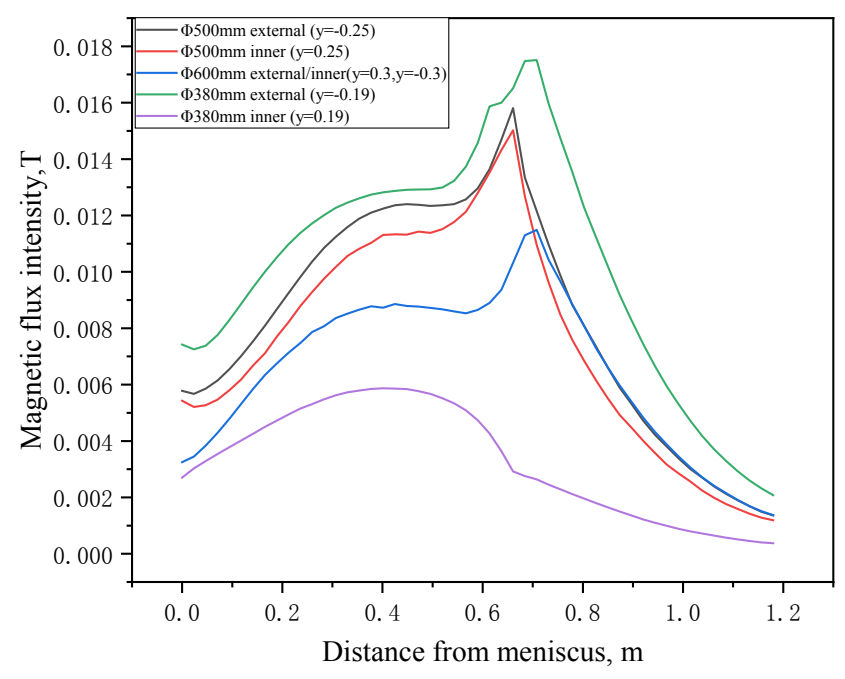

Fig. 5 Magnetic flux density on the external and inner arc sides. the $\$ 380 \mathrm{~mm}$ round bloom, the maximum magnetic flux density is located at the center of M-EMS.

Figure 6 shows the magnetic flux density of the crosssection at the mold exit of the $\Phi 380 \mathrm{~mm}$ round bloom at different times. Although the position of the maximum magnetic flux density changes over time, the magnetic flux density on the external arc side is always greater than that on the inner arc side.

Figure 7 shows the vector and contour plots of the timeaveraged electromagnetic force on the cross section at mold exit of $\Phi 380 \mathrm{~mm}$ round bloom. The electromagnetic force on the external arc side is larger than that on the inner arc side.

\subsection{Flow fields}

Before the solidification shell of the round bloom in mold, the electromagnetic force greatly promotes the velocity of the molten steel. The inertial impingement jets from the nozzle deviate from central axis of the round blooms

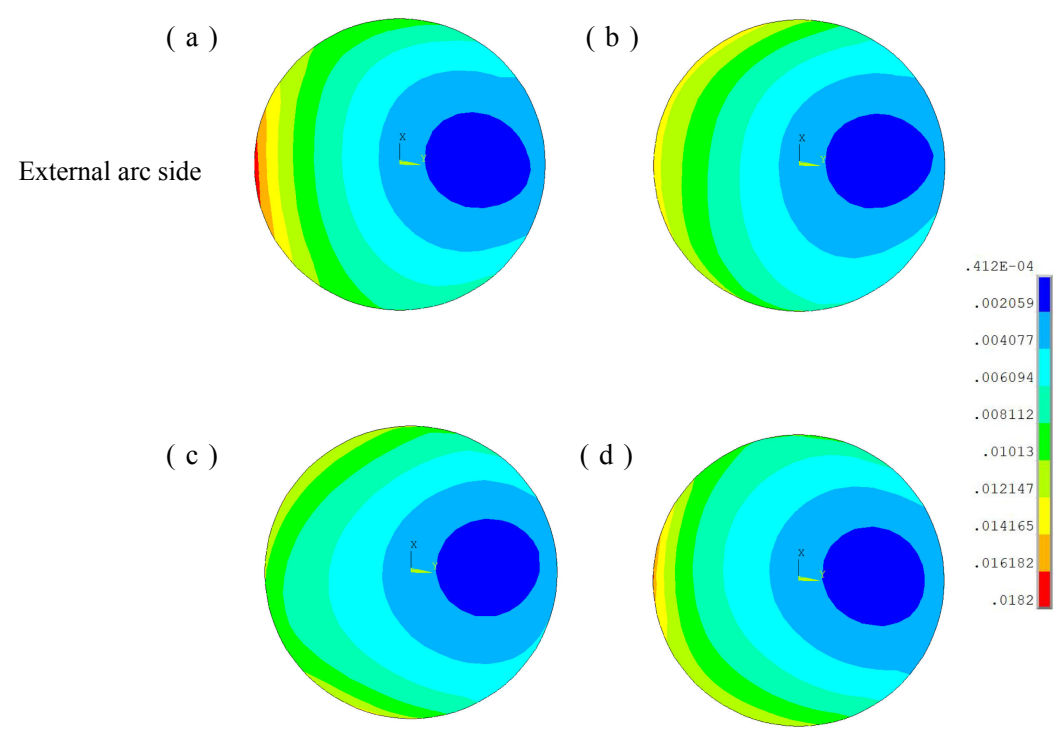

Fig. 6 Magnetic flux density in the cross-profile at the mold exit of the $\Phi 380 \mathrm{~mm}$ round bloom (T). (a) $t=0$, (b) $t=\frac{1}{8} \mathrm{~T}$, (c) $t=\frac{1}{4} \mathrm{~T}$, (d) $\mathrm{t}=\frac{3}{8} \mathrm{~T}$. 


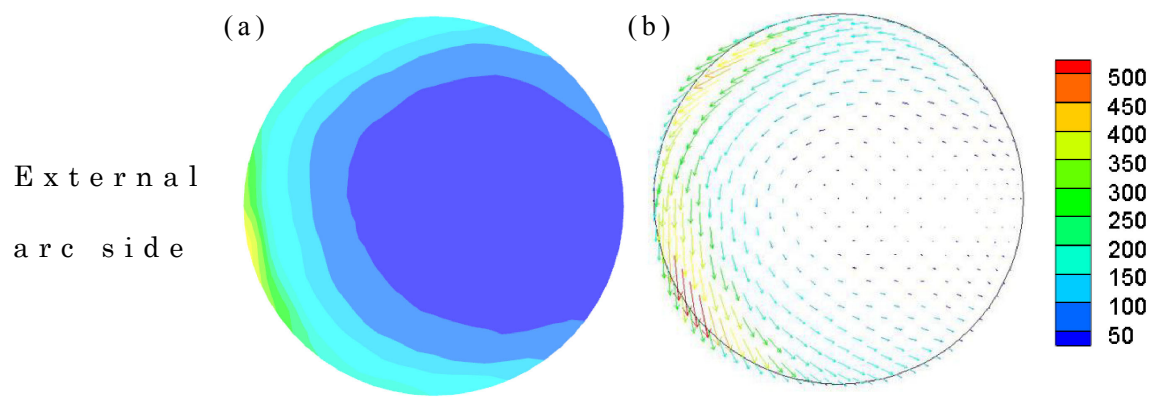

Fig. 7 Electromagnetic force density in the cross profile at mold exit of the $\Phi 380 \mathrm{~mm}$ round bloom (N/m $\mathrm{m}^{3}$ ). (a) Contour plot, (b) vector.
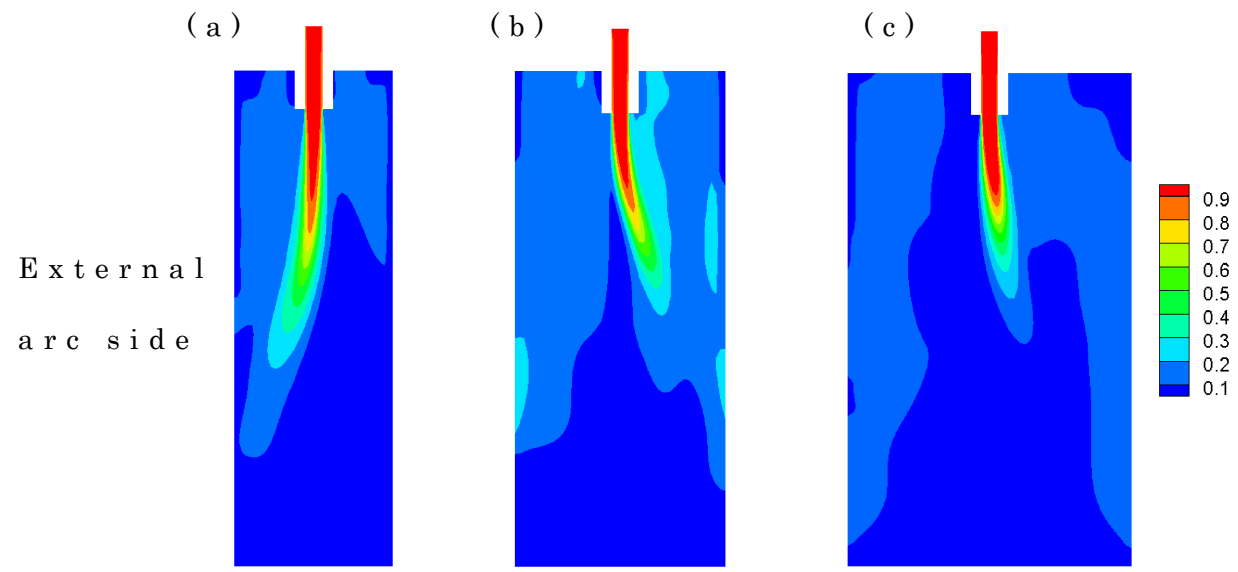

Fig. 8 Flow fields of the round blooms (35 s). (a) $\Phi 380 \mathrm{~mm}$, (b) $\Phi 500 \mathrm{~mm}$, (c) $\Phi 600 \mathrm{~mm}$.

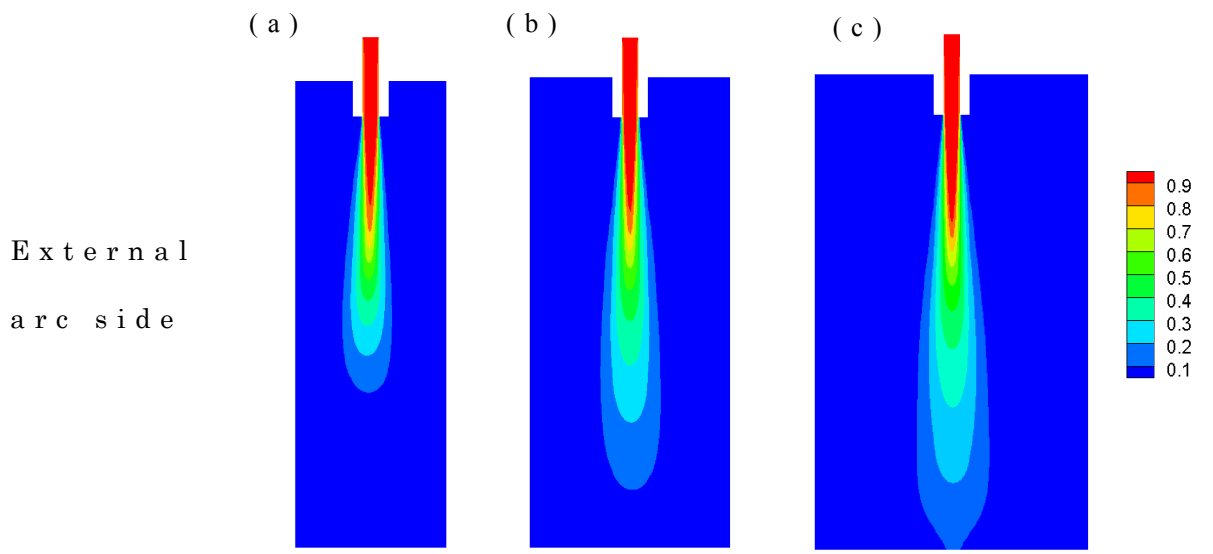

Fig. 9 Flow fields of the round blooms (350 s). (a) $\Phi 380 \mathrm{~mm}$, (b) $\Phi 500 \mathrm{~mm}$, (c) $\Phi 600 \mathrm{~mm}$.

obviously, and the flow fields change periodically. Figure 8 depicts the velocity fields of the profile section of the round blooms at $35 \mathrm{~s}$. These results are similar to those of Ren. ${ }^{11)}$ At this time, the speed of the molten steel at the edge of round bloom is large, and the effects of eccentric stirring on the flow of molten steel of $\Phi 380 \mathrm{~mm}$ and $\Phi 500 \mathrm{~mm}$ round blooms are extremely small.

The formation of shell and the mushy zone of round bloom not only worsens the fluidity of the molten steel at the edges, but also reduces the effect of the M-EMS. As a result, the flow fields of round blooms become stable and no longer change with time. When the calculation time is $350 \mathrm{~s}$, the velocity fields of round blooms are shown in Fig. 9. Figure 10 shows the 2D streamlines of molten steel in the cross-section at the mold exit. Affected by eccentric M-EMS, the electromagnetic force on the external arc side of the $\Phi 380 \mathrm{~mm}$ round bloom is much greater than that on the inner arc side, and the molten steel in the mushy zone inhibits the transfer of momentum. Under the combined action, the inertial impingement jet deflects the external arc side. The electromagnetic force on the external arc side of the $\Phi 500 \mathrm{~mm}$ round bloom is similar to that on the inner arc side, so the inertial impingement jet deflects the external arc side more slightly. The symmetrical electromagnetic force of $\Phi 600 \mathrm{~mm}$ round bloom makes the flow field symmetrical.

\subsection{Temperature fields}

The temperature fields of the profile sections of round blooms are shown in Fig. 11, and the temperatures of the molten steel on the external and inner arc sides of the round 


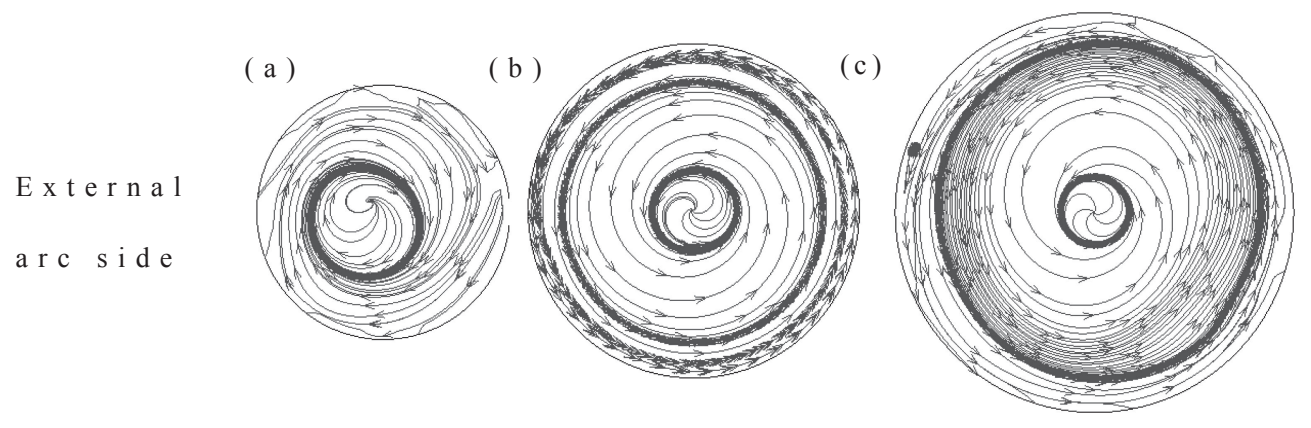

Fig. 10 2D streamlines in cross-section (mold exit). (a) $\Phi 380 \mathrm{~mm}$, (b) $\Phi 500 \mathrm{~mm}$, (c) $\Phi 600 \mathrm{~mm}$.

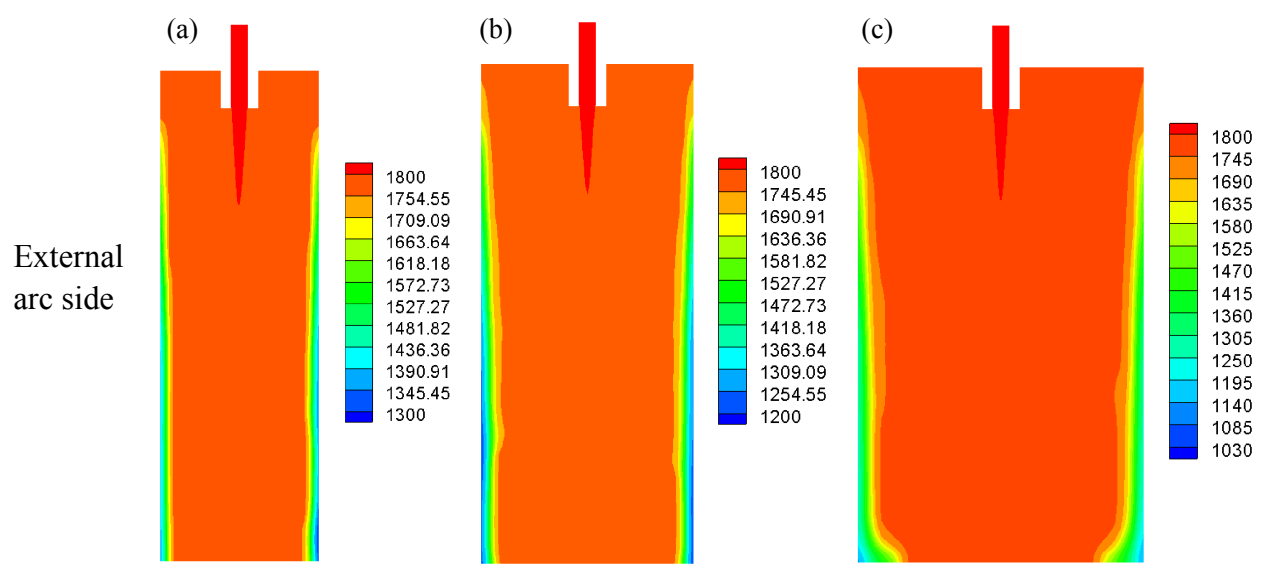

Fig. 11 Temperature fields in the profile section (K). (a) $\Phi 380 \mathrm{~mm}$, (b) $\Phi 500 \mathrm{~mm}$, (c) $\Phi 600 \mathrm{~mm}$.

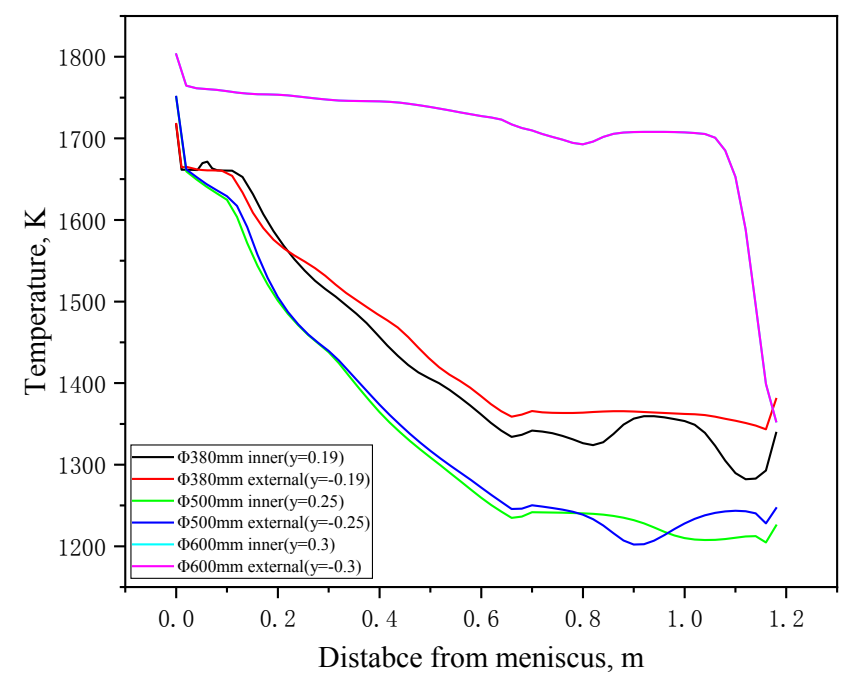

Fig. 12 Temperature of the molten steel on the external and inner arc sides at the foot roller exit.

blooms are shown in Fig. 12. The mushy zone of the $\Phi 600 \mathrm{~mm}$ round bloom is the largest among all the simulated diameters since its lowest casting speed. Compared with the $\Phi 600 \mathrm{~mm}$ round bloom, the inertial impingement jets of the $\Phi 380 \mathrm{~mm}$ and $\Phi 500 \mathrm{~mm}$ round bloom deflect their external arc side because of the eccentric M-EMS effect. This makes the temperature of the molten steel higher in the external arc side. From the simulation results, the maximum temperature difference of the $\Phi 380 \mathrm{~mm}$ and $\Phi 500 \mathrm{~mm}$ round bloom are $69 \mathrm{~K}$ and $33 \mathrm{~K}$, respectively.

\section{Effects of the Nozzle Position}

The flow and temperature fields in the mold affect the solidification and solute transport of continues casting billets. ${ }^{18)}$ The simulation results on the temperature field shows that the eccentric M-EMS makes a higher temperature of molten steel on the external arc side, which weaken the thickness of the shell and increases the possibility of cracking. Therefore, it is necessary to decrease the temperature of the molten steel on the external arc side.

The idea of this research is to move the nozzle from the inner arc side by $50 \mathrm{~mm}$ in orders to bring the high temperature molten steel from the nozzle closer to the inner arc side of the round bloom to compensate the eccentric M-EMS effect. The new geometric model is shown in Fig. 13. The only change of the new geometric model to the previous one is the position of the nozzle.

Figure 14(a) and (b) show the 2D streamlines of round blooms with central and modified nozzle positions. When the nozzle is at the center of the round bloom, the impinging jet from the nozzle produces 2 recirculation, the recirculation zone on the external arc side is smaller, but closer to the edge of the round bloom. To the nozzle modified position, the molten steel entering the mold flows to the inner arc side, and an extremely large recirculation zone is produced on the external arc side only.

The flow field of the profile section of the round bloom with modified nozzle position is shown in Fig. 15(a), and the corresponding temperature field is shown in Fig. 15(b). The flow field shows that the molten steel entering the mold flows 


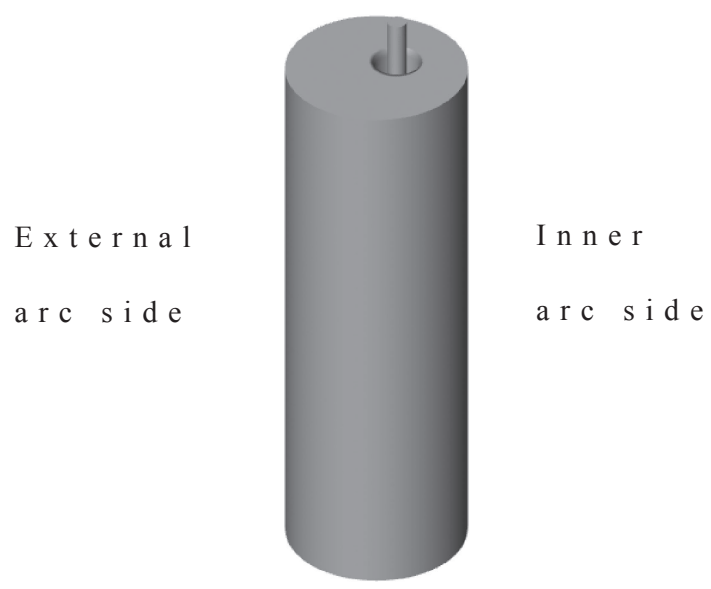

Fig. 13 New geometric model.
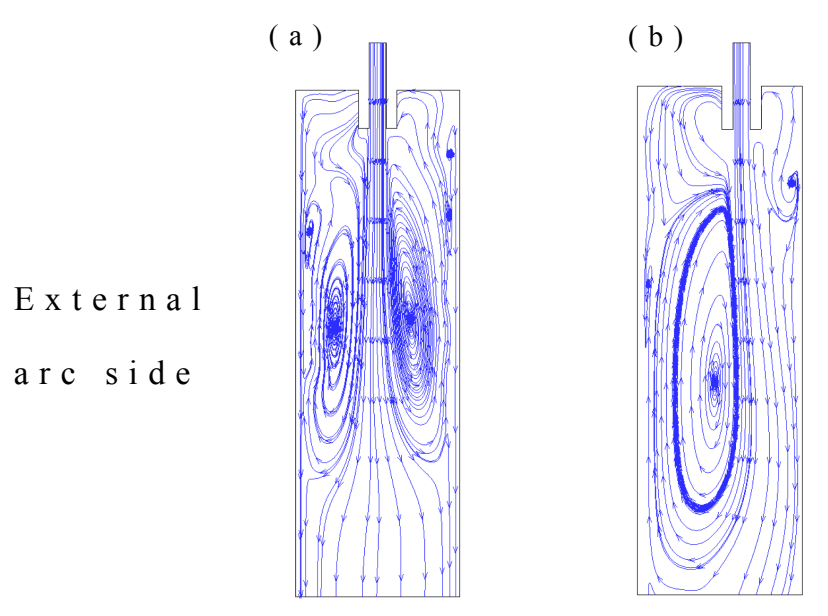

Fig. $142 \mathrm{D}$ streamlines in the profile section. (a) Nozzle at the center, (b) nozzle near the inner arc side.

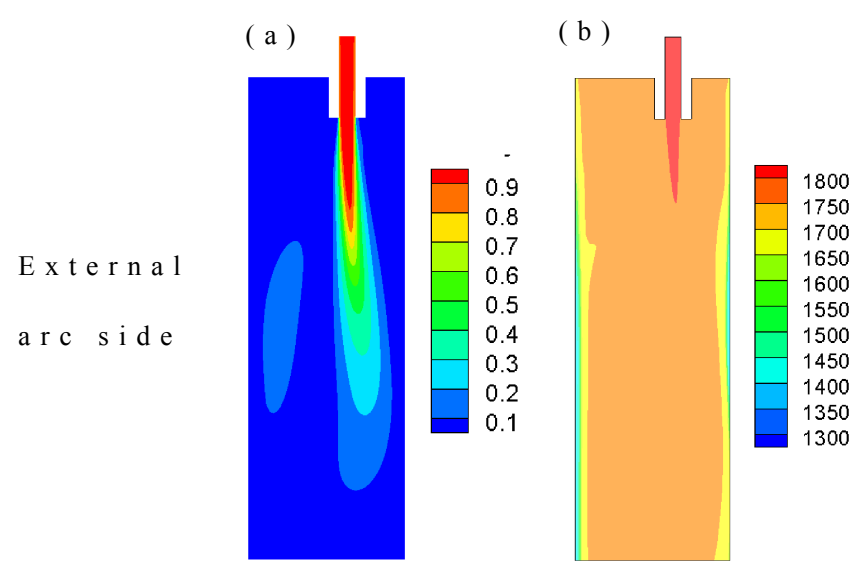

Fig. 15 The flow and temperature fields of round bloom in the profile section. (a) Flow field, (b) temperature field.

slightly toward the inner arc side. Consisting with flow field of the round bloom, the temperature of the molten steel on the external arc side is smaller than that at inner arc side. This indicates that the inertial impingement jet is mainly affected by the electromagnetic force near the inner arc side, and the greater electromagnetic force at the sides makes the molten steel flow to the inner arc side.

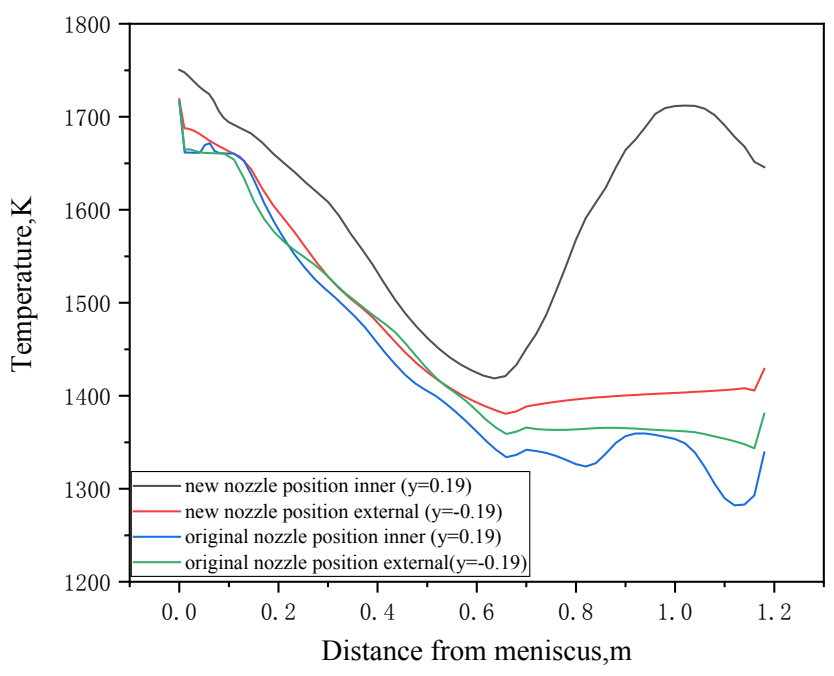

Fig. 16 Temperature of molten steel on the external and inner arc sides at the foot roller exit.

The temperature on the external and inner arc sides of the round bloom are shown in Fig. 16. A comparison of Fig. 16 with Fig. 12 shows that with modified nozzle position, the temperature of molten steel on the external arc side decreases, which is in line with the expected idea. However, the temperature of the molten steel on the inner arc side increase obviously, which increases the possibility cracking.

The above results show that moving the nozzle to the inner arc sides is beneficial to decrease the molten steel temperature on the external arc side but substantially increases the molten steel temperature on the inner arc side. Although the unevenness of the temperature still exists, the modification of nozzle position still meaningful since it provides a method to compensate the effect of eccentric M-EMS in the round bloom. It can be predicted that by increasing the M-EMS current intensity and reducing the offset distance of the nozzle, the temperature difference of the molten steel between the inner and external arc can be further alleviated, and thus reducing the effect of eccentric M-EMS. In future work, we plan to study the effects of the nozzle position and M-EMS parameters on the $\Phi 380 \mathrm{~mm}$ round bloom.

\section{Conclusion}

A coupled 3D mathematical model is established to study the electromagnetic, flow and temperature fields of round blooms with different degrees of eccentric M-EMS. The conclusions are as follows:

(1) Under the conditions of eccentric M-EMS, the magnetic flux density and time-averaged electromagnetic force on the external arc side of the round bloom are greater than those on the inner arc side, and these trends do not change over time. Under severe eccentric M-EMS, the maximum value of the magnetic flux density on the inner arc side of the $\Phi 380 \mathrm{~mm}$ round bloom is located at the center of M-EMS, while the maximum value of the magnetic flux density on the external arc side is located at the exit of the mold.

(2) When the solidified shell is not yet formed, the flow fields of the round blooms change periodically under 
the action of M-EMS. As the solidification progresses, the flow field of the molten steel gradually stabilizes. Due to the greater electromagnetic force on the external arc side, the inertial impingement jets of the $\Phi 380 \mathrm{~mm}$ and $\Phi 500 \mathrm{~mm}$ round blooms ultimately deviate slightly toward the external arc sides.

(3) Under the effects of eccentric M-EMS, the temperature of the molten steel on the external arc side of the $\Phi 380 \mathrm{~mm}$ round bloom is higher than that on the inner arc side, and the maximum temperature difference is $69 \mathrm{~K}$.

(4) After the nozzle position of the $\Phi 380 \mathrm{~mm}$ round bloom is moved toward the inner arc side by $50 \mathrm{~mm}$, the temperature of the molten steel on the external arc side decreases, but the temperature of the molten steel on the inner arc side increases significantly. The nozzle position and M-EMS parameters need to be further studied to mitigate the impact of eccentric M-EMS.

\section{Acknowledgments}

This research was funded by the National Natural Science Fund of China, grant number 51674185.

\section{REFERENCES}

1) H. Sun and J. Zhang: Metall. Mater. Trans. B 45 (2014) 1133-1149.

2) L. Cai, X. Wang and N. Wang: Mater. Trans. B 51 (2020) 236-246.

3) M.J. Li, Y. Murakami, I. Matsui, N. Omura and S.J. Tada: Mater. Trans. 59 (2018) 1603-1609.

4) Y. Zhai, K. Pan and D. Wu: Metals 9 (2019) 993-1005.

5) D. Schurmann, B. Willers and G. Hackl: Mater. Trans. B 50 (2019) 716-731.

6) H. Wu, N. Wei and Y. Bao: Int. J. Miner. Metall. Mater. 18 (2011) 159164.

7) S.I. Shakhov and K.N. Vdovin: Steel Transl. 49 (2019) 261-264.

8) D. Jiang and M. Zhu: Mater. Trans. B. 47 (2016) 3446-3458.

9) Z.H. Zhang, J.L. Jun and W.P. Liu: Steelmaking 29 (2013) 19-22.

10) W. Su, M.Y. Zhu and Z.Q. Zhang: Continue Casting 3 (2009) 42-47.

11) B.Z. Ren, D.F. Chen, H.D. Wang and H. Wang: Steel Res. Int. 86 (2015) 1104-1115.

12) M.R.R.I. Shamsi and S.K. Ajmani: ISIJ Int. 47 (2007) 433-442.

13) M.R. Aboutalebi, M. Hasan and R.I.L. Guthrie: Mater. Trans. B 26 (1995) 731-744.

14) M.Y. Ha, H.G. Lee and S.H. Seong: J. Mater. Process. Technol. 133 (2003) 322-339.

15) P.T. Hietanen, S. Louhenkilpi and S. Yu: Steel Res. Int. 88 (2017) 1600355.

16) H. Yang, X. Zhang, K. Deng, W. Li, Y. Gan and L. Zhao: Mater. Trans. B 29 (1998) 1345-1356.

17) Q. Fang, H.W. Ni and S.J. Wang: Steelmaking 30 (2014) 57-61.

18) W. Zhang, S. Luo, Y. Chen, W. Wang and M. Zhu: Metals 9 (2019) 6684. 